\title{
The changing clinical spectrum of liver abscess: the Jerusalem experience
}

\author{
A.M. Yinnon, I. Hadas-Halpern ${ }^{1}$, M. Shapiro ${ }^{2}$ and C. Hershko ${ }^{3}$ \\ Infectious Diseases Unit, Departments of ${ }^{1}$ Radiology and ${ }^{3}$ Medicine, Shaare Zedek Medical Center, \\ Jerusalem, and ${ }^{2}$ Department of Clinical Microbiology and Infectious Diseases, Hadassah Hebrew \\ University Medical Center, Jerusalem 91031, Israel
}

Summary: A retrospective study was conducted of all 31 patients with liver abscess admitted to Shaare Zedek Medical Center between 1979, the year computed tomographic scan and ultrasound were introduced, and 1992. Fever and abdominal pain were the most common symptoms. Duration of symptoms was short: $81 \%$ of patients had symptoms for $\leqslant 5$ days and none had symptoms for more than 3 weeks. Amoebic abscess was found in one patient only, the remainder were pyogenic. Biliary tract pathology remains the most common cause of liver abscess (39\%). The diagnosis was made by ultrasound in $22 / 31$ (71\%) and by computed tomographic scan in $9 / 31$ (29\%) of patients. Treatment consisted of intravenous antibiotics and percutaneous drainage under ultrasound guidance in $24 / 31$ patients (77\%). Four patients died $(13 \%)$, three of whom had underlying malignancies.

Clinical features were compared with those from a similar series of 36 cases with liver abscess reported from Jerusalem predating ultrasound and computed tomographic scan (1967-1977). Several major changes have occurred. First, the relative incidence of amoebic abscess has dramatically decreased. Second, ultrasound and computed tomographic scan have facilitated earlier diagnosis and percutaneous drainage, contributing to improved survival and lower morbidity.

\section{Introduction}

During the last decade, the diagnosis and management of pyogenic liver abscess have changed significantly. ${ }^{1}$ The introduction of computed tomographic (CT) scan and ultrasound in the late 1970 s and early 1980 s has enabled earlier diagnosis and percutaneous drainage. This report reviews the experience with liver abscess in one medical center in Jerusalem after modern imaging techniques were made available.

A computer-assisted search was performed for all patients who received the discharge diagnosis of liver abscess at Shaare Zedek Medical Center after 1979, the year CT scan was introduced in Shaare Zedek Medical Center. The diagnosis was based on characteristic clinical, ultrasonographic or CT scan findings. Duration of symptoms was defined as the time from the first symptoms attributable to abscess to time of admission. Patient characteristics analysed included age, sex, duration and nature of symptoms, physical findings, laboratory results, diagnostic tools, site and number of abscesses, treatment and outcome.

We wished to assess the changes in aetiology, diagnosis and management of liver abscess that

Correspondence: A.M. Yinnon, M.D.

Accepted: 3 December 1993

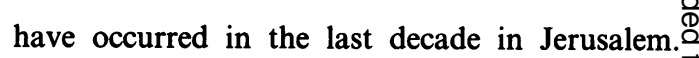
Therefore, data from the present series were com- $\vec{\Rightarrow}$ pared with data from a similar series of patients 3 consecutively admitted to the Hadassah University Medical Center, Jerusalem, Israel between 1967 우․ and $1977 .^{2}$

Results

Thirty-one patients were diagnosed with liver abscess at Shaare Zedek Medical Center between옹 1979 and 1992; there were 18 men and 13 women, ranging in age from 5 to 91 years (mean $55 \pm 25$ ). $A$ ․ total of 23 patients were Jewish and eight Arab. N Duration of symptoms prior to admission was $N$ relatively short: 25 patients $(81 \%)$ had symptoms for 5 days or less, four patients $(13 \%)$ had sym- $\omega$ ptoms for 6-14 days, and only two patients had symptoms for more than 2 weeks. Duration of admission was $39 \pm 22$ days (mean \pm s.d.).

Table I presents the symptoms and signs of the $\stackrel{?}{?}$ 31 patients. Biliary tract pathology remains the $\frac{0}{0}$ commonest underlying cause of liver abscess $(12 \stackrel{\mathrm{D}}{\mathrm{D}}$ patients, $39 \%$ ): eight patients had acute cholecyst- $\frac{?}{+}$ itis, two patients had prior choledocho-duodeno- $\stackrel{\perp}{\circ}$ stomy, one patient had cholangiocarcinoma and one patient had a recent cholecystectomy. 
Table I Symptoms and signs of patients with liver abscess

\begin{tabular}{lrl}
\hline Symptom/sign & Number of patients $(\mathrm{n}=31)$ \\
\hline Abdominal pain & 23 & $(74 \%)$ \\
Fever & 20 & $(65 \%)$ \\
Nausea & 15 & $(48 \%)$ \\
Malaise/weakness & 10 & $(39 \%)$ \\
Vomiting & 9 & $(29 \%)$ \\
Jaundice & 5 & $(16 \%)$ \\
Pulmonary oedema & 4 & $(13 \%)$ \\
Haematemesis & 3 & $(10 \%)$ \\
Shock & 3 & $(10 \%)$ \\
Stupor & 3 & $(10 \%)$ \\
\hline
\end{tabular}

Drainage of infection through the portal venous system caused liver abscess in four patients $(13 \%)$ : two patients had recent appendicectomy, one patient had recent sigmoidectomy for carcinoma, and one patient had diverticulitis. Contiguous spread precipitated abscess formation in five patients $(16 \%)$ : four patients had sustained abdominal trauma and another patient had recent resection of a hydatid cyst in the liver. One patient had amoebic abscess, diagnosed by serial serological testing and one other abscess was of haematogenous origin. No underlying aetiology could be determined in eight patients $(26 \%)$.

Thirty of the 31 patients $(97 \%)$ had a pyogenic liver abscess. Thirty patients underwent percutaneous aspiration. Table II shows the organisms cultured from aspirated pus. Non-specific laboratory tests were generally not helpful. Haemoglobin was $>12 \mathrm{~g} / \mathrm{dl}$ in $45 \%$ of patients, and moderate anaemia (haemoglobin $<10 \mathrm{~g} / \mathrm{dl}$ ) occurred in only $26 \%$ of cases. The white blood count was $<10.0 \times 10^{9} / 1$ in $29 \%$ and $>15 \times 10^{9} / 1$ in $32 \%$ of patients. Alanine aminotransferase was above normal levels in $55 \%$ of patients, aspartate aminotransferase in $54 \%$, lactic dehydrogenase in $83 \%$, alkaline phosphatase in $56 \%$ and bilirubin in $60 \%$. All five liver function tests were normal in three patients only.

The diagnosis was made by ultrasound in $22 / 31$ $(71 \%)$ and by CT scan in $9 / 31(29 \%)$ of patients. A single abscess was found in 23 patients $(74 \%)$, multiple abscesses were diagnosed in eight patients $(26 \%)$. Most abscesses were located in the right lobe ( 20 patients, $64 \%$ ); in five patients the abscess was in the left lobe, in three patients in both lobes. In three patients films were unavailable for review.

Treatment modalities are shown in Table III. Four patients died (13\%); their underlying diseases included (one patient in each category): cholangiocarcinoma, sigmoid carcinoma with operative complications, cholecystitis with empyema, and agnogenic myeloid metaplasia treated with steroids. Overall survival was $\mathbf{8 7 \%}$.

In Table III a comparison is made between the clinical features of patients with liver abscess in the current study with those from a similar series reported from Jerusalem. ${ }^{2}$

\section{Discussion}

In 1938 Ochsner published the then largest series of liver abscess. ${ }^{3}$ That series included 139 cases of amoebic abscess and 47 patients with pyogenic abscess and underlined the uniform mortality of patients treated non-operatively. Subsequent series have emphasized the changing clinical patterns of liver abscess even before the advent of modern imaging techniques. ${ }^{4,5}$ The overall incidence of the disease has remained stable during the last five decades. ${ }^{6,7}$

The importance of biliary and colonic disease as an underlying cause is evident in our series, with $52 \%$ of patients with these sites as the source of infection. Despite intensive investigations in most cases, $26 \%$ of the patients had no underlying source identified. Ultrasound and CT scan have had a profound impact on the management and outcome of liver abscess. ${ }^{8,9}$ In the present series a majority of patients $(77 \%)$ underwent percutaneous drainage. Surgical therapy sometimes remains necessary for abscesses of the left lobe or those that are multi-loculated, or when this approach is advisable because of concomitant intra-abdominal disease. In our series no difference was found in outcome between patients managed medically or surgically. The overall survival of patients with liver abscess was $87 \%$, substantially higher than in the pre-antibiotic era, ${ }^{3}$ but also significantly higher than in the period immediately before the introduction of CT scan and ultrasound. ${ }^{2,4}$ Currently, underlying malignancy appears to be the most important determinant of outcome of liver abscess; three of the four patients who died in our series had malignant diseases.

Duration of symptoms before establishment of diagnosis appears nowadays to be relatively short; this may be due to early diagnosis with ultrasound or CT scan, but could also reflect that a greater percentage of patients have pyogenic abscess producing a more acute presentation than amoebic abscess. Both ultrasound and CT scan are highly sensitive to abnormality, although the literature continues to indicate a group of patients diagnosed only by operation or autopsy. ${ }^{10}$

Recent reviews emphasize the evolving microbiology of liver abscess. ${ }^{11}$ Amoebic abscess, once common in Jerusalem and still prevalent in many developing countries, appears to be disappearing in the western world. ${ }^{12,13}$ Modern culture techniques 
Table II Abscess culture results

\begin{tabular}{lrc}
\hline Cultured organism & Number of & organisms (n = 55) \\
\hline Aerobic Gram-negative bacilli & 30 & $(54 \%)$ \\
Klebsiella pneumoniae & 7 & \\
Escherichia coli & 6 & \\
Pseudomonas aeruginosa & 5 & \\
Serratia marcescens & 4 & \\
Proteus mirabilis & 3 & \\
Morganella morganii & 2 & \\
Citrobacter freundii & 2 & \\
Salmonella & 1 & \\
Aerobic Gram-positive cocci & 16 & $(29 \%)$ \\
Enterococcus & 6 & \\
Staphylococcus aureus & 5 & \\
Staphylococcus epidermidis & 2 & \\
Alpha-haemolytic Streptococcus & 3 & \\
Anaerobic organisms* & 9 & $(15 \%)$ \\
Bacteroides fragilis & 6 & \\
Sarcina & 2 & \\
Clostridium species & 1 & \\
Total & $55^{*}$ & \\
\hline
\end{tabular}

*30/31 patients had their abscess(es) aspirated: seven were sterile. Ten of the 23 positive cultures grew one organism, 13 grew $\geqslant$ two organisms.

Table III Liver abscess: the changes in aetiology, diagnosis, management and outcome in Jerusalem

\begin{tabular}{|c|c|c|c|}
\hline Clinical parameter & $\begin{array}{c}1979-1992^{*} \\
(\mathrm{n}=31)\end{array}$ & $\begin{array}{c}1967-1977 \dagger \\
(\mathrm{n}=36)\end{array}$ & $\mathbf{P} \ddagger$ \\
\hline $\begin{array}{l}\text { Symptoms/signs } \\
\text { Fever }>37.6^{\circ} \mathrm{C} \\
\text { Malaise/weakness } \\
\text { Vomiting } \\
\text { Loss of weight } \\
\text { Shortness of breath } \\
\text { Duration of symptoms } \$\end{array}$ & $\begin{aligned} 20 & (65 \%) \\
12 & (39 \%) \\
9 & (29 \%) \\
2 & (6 \%) \\
2 & (6 \%) \\
6.4 \pm & 3.5 \text { days }\end{aligned}$ & $\begin{array}{cl}34 & (94 \%) \\
33 & (92 \%) \\
21 & (58 \%) \\
15 & (42 \%) \\
12 & (33 \%) \\
3.2 \pm & \pm .9 \text { months }\end{array}$ & $\begin{array}{l}<0.01 \\
<0.001 \\
<0.05 \\
<0.001 \\
<0.001 \\
<0.001\end{array}$ \\
\hline $\begin{array}{l}\text { Aetiology } \\
\text { Amoebic abscess } \\
\text { Pyogenic abscess } \\
\text { Anaerobic organisms } \pi\end{array}$ & $\begin{aligned} 1 & (3 \%) \\
30 & (97 \%) \\
5 & (15 \%)\end{aligned}$ & $\begin{aligned} 15 & (42 \%) \\
21 & (58 \%) \\
0 & \end{aligned}$ & $\begin{array}{l}<0.01 \\
<0.01 \\
<0.01\end{array}$ \\
\hline $\begin{array}{l}\text { Diagnosis } \| \\
\text { Non-invasive procedure } \\
\text { Surgical diagnosis } \\
\text { Post-mortem diagnosis }\end{array}$ & $\begin{aligned} 31 & (100 \%) \\
0 & \\
0 & \end{aligned}$ & $\begin{aligned} 21 & (58 \%) \\
9 & (25 \%) \\
6 & (17 \%)\end{aligned}$ & $\begin{array}{l}<0.001 \\
<0.01 \\
<0.01\end{array}$ \\
\hline $\begin{array}{l}\text { Management** } \\
\text { Percutaneous drainage only } \\
\text { Percutaneous and open drainage } \\
\text { Open drainage only } \\
\text { Antimicrobials only }\end{array}$ & $\begin{aligned} 24 & (77 \%) \\
5 & (16 \%) \\
1 & (3 \%) \\
1 & (3 \%)\end{aligned}$ & $\begin{aligned} 2 & (5 \%) \\
0 & \\
18 & (50 \%) \\
10 & (28 \%)\end{aligned}$ & $\begin{array}{l}<0.001 \\
<0.01 \\
<0.001 \\
<0.01\end{array}$ \\
\hline Survival & 27 (87\%) & $22 \quad 61 \%)$ & $<0.01$ \\
\hline
\end{tabular}

${ }^{*}$ Present series. †Previously reported series. ${ }^{2} \ddagger$ Student's $t$-test. §Duration of symptoms ranged from 1 to 18 days in the present series as compared to 3 days to 13 months in the early 1970s. TIn the present series $15 / 55$ organisms isolated from the abscess were anaerobes, as compared to $0 / 18$ in the 1970s. |Non-invasive procedure: ultrasound and CT scan in current series, liver-spleen scan in previous series. ${ }^{* *}$ All patients received antimicrobial therapy. 
frequently lead to isolation of anaerobic organisms, often in addition to aerobic Gram-negative bacilli and Gram-positive cocci.

The comparison of case series has certain drawbacks. However, the two hospitals reporting these series serve the same population, have identical case mixes and employ similar approaches. We therefore believe that the comparison of these series does provide valid insights in the evolving clinical picture of liver abscess in Jerusalem.

\section{References}

1. Frey, C.F., Zhu, Y., Suzuki, M. \& Isaji, S. Liver abscesses. Surg Clin North Am 1989, 69: 259-271.

2. Shapiro, M., Rachmilewitz, D., Avinoah, I., Katz, S. \& Bar-Sela, S. Liver abscess at the Hadassah University Hospital during the years 1967-77. Isr J Med Sci 1980, 16: 761-766.

3. Ochsner, A., DeBakey, M. \& Murray, S. Pyogenic abscess of the liver. Am J Surg 1938, 40: 292-314.

4. Lee, J.F. \& Block, G.E. The changing clinical pattern of hepatic abscesses. Arch Surg 1972, 104: 465-470.

5. Rubin, R.H., Swartz, M.N. \& Malt, R. Hepatic abscess: changes in clinical, bacteriologic and therapeutic aspects. $\mathrm{Am}$ J Med 1974, 57: 601-610.

6. Gyorffy, E.J., Frey, C.F., Silva, J. Jr \& McGahan, J. Pyogenic liver abscess: diagnostic and therapeutic strategies. Ann Surg 1987, 206: 699-705.

7. Heymann, A.D. Clinical aspects of grave pyogenic abscesses of the liver. Surg Gynecol Obstet 1979, 149: 209-213.

8. Klatchko, B.A. \& Schwartz, S.I. Diagnostic and therapeutic approaches to pyogenic abscess of the liver. Surg Gynecol Obstet 1989, 168: 332-336.

\section{Acknowledgement}

The authors thank Ms Hana Cohen, Head of Medical Archives, Shaare Zedek Medical Center, for her kind and professional assistance in locating and retrieval of medical records.

9. Farges, O., Leese, T. \& Bismuth, H. Pyogenic liver abscess: an improvement in prognosis. Br J Surg 1988, 75: 862-865.

10. Satiani, B. \& Davidson, E.D. Hepatic abscesses: improvement in mortality with early diagnosis and treatment. $\mathrm{Am} \mathrm{J}$ Surg 1978, 135: 647-650.

11. McDonald, M.I., Corey, G.R. \& Gallis, H.A. Single and multiple pyogenic liver abscesses. Natural history, diagnosis and treatment, with emphasis on percutaneous drainage. Medicine 1984, 63: 291-302.

12. Donovan, A.J., Yellin, A.E. \& Ralls, P.W. Hepatic abscess. World J Surg 1991, 15: 162-169.

13. Barnes, P.F., De Cock, K.M., Reynolds, T.N. \& Ralls, P.W. A comparison of amebic and pyogenic abscess of the liver. Medicine 1987, 66: 472-483.

14. Bertel, C.K., van Heerden, J.A. \& Sheedy, P.F. Treatment of pyogenic hepatic abscesses: surgical vs percutaneous drainage. Arch Surg 1986, 121: 554-558. 